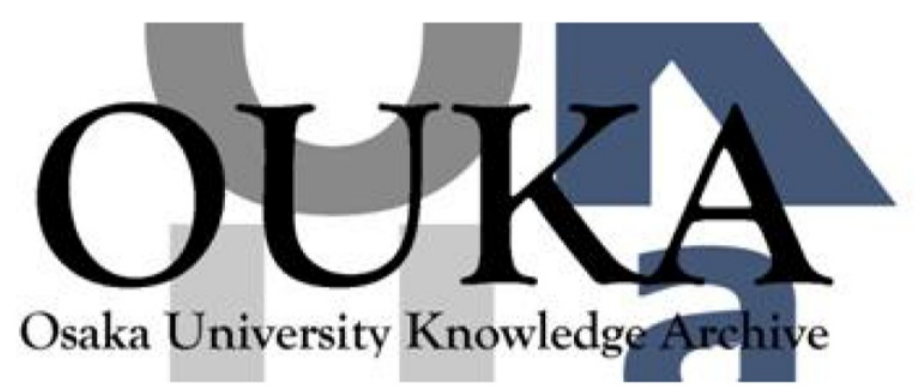

\begin{tabular}{|c|c|}
\hline Title & $\begin{array}{l}\text { Formation of continuous metallic film on quartz } \\
\text { studied by noncontact resonant ultrasound } \\
\text { spectroscopy }\end{array}$ \\
\hline Author (s) & Nakamura, N.; Yoshimura, N.; Ogi, H. et al. \\
\hline Citation & $\begin{array}{l}\text { Journal of Applied Physics. 118(8) p.085302-1- } \\
\text { p. } 085302-6\end{array}$ \\
\hline Issue Date & $2015-08-24$ \\
\hline oaire:version & VoR \\
\hline URL & https://hdl. handle. net/11094/83918 \\
\hline rights & $\begin{array}{l}\text { Copyright } 2015 \text { AIP Publishing LLC. This article } \\
\text { may be downloaded for personal use only. Any } \\
\text { other use requires prior permission of the } \\
\text { author and AIP Publishing. This article } \\
\text { appeared in Journal of Applied Physics, 118(8), } \\
085302,2015 \text { and may be found at } \\
\text { https://doi.org/10.1063/1.4928959. }\end{array}$ \\
\hline Note & \\
\hline
\end{tabular}

Osaka University Knowledge Archive : OUKA

https://ir. Library. osaka-u. ac. jp/

Osaka University 


\title{
Formation of continuous metallic film on quartz studied by noncontact resonant ultrasound spectroscopy
}

\author{
N. Nakamura, ${ }^{\text {a) }}$ N. Yoshimura, H. Ogi, and M. Hirao \\ Osaka University, Graduate School of Engineering Science, Toyonaka, Osaka 560-8531, Japan
}

(Received 26 May 2015; accepted 8 August 2015; published online 24 August 2015)

\begin{abstract}
Dynamics of continuous film formation of metallic films on quartz substrates is studied using an electrodeless resonance method. Bare quartz is used as a substrate, and a metallic film is deposited on it. We use antenna transmission technique to measure the evolution of resonance frequencies and internal friction of the substrate during and after deposition, and the morphological transition between discontinuous islands and a continuous film is detected. By comparison with atomic force microscopy images, we confirm that the frequency drop and the internal-friction peak that appear during deposition indicate this transition. We also find that $\mathrm{Pt}$ film shows unexpected morphology change after deposition. (C) 2015 AIP Publishing LLC.
\end{abstract}

[http://dx.doi.org/10.1063/1.4928959]

\section{INTRODUCTION}

The formation of metallic films on substrates during deposition is often explained by the Volmer-Weber growth, in which continuous films are formed through the growth of isolated islands and their percolation. The transition between discontinuous and continuous structures causes considerably changes in the electric conductivity, ${ }^{1}$ magnetic coercivity, ${ }^{2}$ and elastic stiffness. ${ }^{3,4}$ Morphology evolution occurs also after deposition. The evolution behavior varies depending on the interrupted time. ${ }^{5,6}$ Monitoring the morphology change during and after deposition is an important issue for understanding the dynamics of film growth and for controlling the deposition to produce films with desired properties.

Electric resistance measurements are a typical method for investigating the morphology change of thin film during and after deposition. ${ }^{1,5,7-10}$ In the early stages of deposition, the tunneling current between isolated islands contributes to the surface conduction, and the surface resistance decreases greatly as the inter-island distance decreases with increasing the island size. ${ }^{7}$ Once a continuous film has been formed, the decrement rate of resistance becomes smaller. Curvature measurements provide another way to evaluate the morphology change. Intrinsic stress in the deposited structure is evaluated from the curvature of the substrate, and the evolution of the deposited structure is evaluated from the stress evolution. ${ }^{11-14}$ For example, an increment in the tensile stress indicates island coalescence. By using these methods, the morphology change from discontinuous to continuous structure is detectable during deposition; in the resistance measurement, the change in resistance becomes moderate once a continuous film is formed, and in the curvature measurement, the increment in the tensile stress indicates the percolation of islands. However, neither the electric resistance nor the intrinsic stress is sensitive to the moment of the transition to a continuous film.

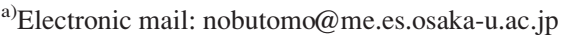

In contrast with these methods, surface acoustic wave (SAW) measurement ${ }^{15-18}$ gives clear indication of the morphology change; it is detected from the attenuation peak. However, the SAW measurement requires electrodes to be located directly on piezoelectric substrates for transmission and detection of SAW.

In this study, we develop an alternative acoustic method. It measures resonance frequencies and internal friction of mechanical free vibration of a naked piezoelectric material without attaching any electrodes. Using this method, we investigate the formation of $\mathrm{Ag}, \mathrm{Cu}$, and $\mathrm{Pt}$ films on quartz substrates, and it is revealed that the morphology evolution during and after deposition can be analyzed by considering the relationship between resonance frequency and internal friction. Finally, we show that ultra-thin Pt on quartz exhibits unexpected morphology changes after deposition.

\section{EXPERIMENTAL SETUP}

Resonance vibration of a piezoelectric rectangular parallelepiped is measured. When a mass is attached to a piezoelectric material, the resonance frequency of the piezoelectric material decreases with increasing mass. This relationship between resonance frequency and mass has already been used to build deposition-rate sensors and biosensors. ${ }^{19-21}$ In addition to measuring the increase in mass, it is also possible to measure the conductivity of the adsorbed material, because changes in the electrical boundary conditions also affect the resonance vibrations of piezoelectric materials. Because the morphology change of metallic film affects the electric boundary conditions, it would be detectable by monitoring the resonance vibrations. However, conventional quartz oscillators cannot detect this morphology change, because their surfaces are covered with metallic electrodes and adsorption of atoms on the electrodes hardly changes the electric boundary conditions. To detect the changes in the boundary conditions, here we use an electrodeless resonance technique. ${ }^{22,23}$ This technique allows us to oscillate a naked quartz substrate without electrodes by using 


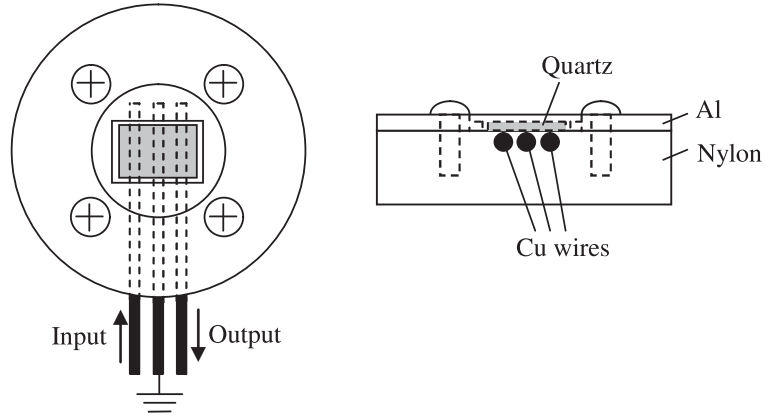

FIG. 1. Schematic of the antennas. Top (left) and cross sectional (right) view.

noncontacting antennas, which are installed in the sputtering chamber. Resonance vibrations are therefore affected by conductivity of deposited films as well as the mass.

Three line antennas are embedded in a nylon base (Fig. 1). ${ }^{22}$ An Al plate with a square-shaped hole is attached to the base, and the quartz substrate is placed in the hole. One of the antennas is grounded and sandwiched between the antennas that are used for transmission and detection of the resonance vibrations. Tone-bursts signals with amplitude of $20 \mathrm{~V}_{\mathrm{pp}}$ are applied to the transmission antenna, and the tangential electric field between the transmitting antenna and the ground antenna excites vibrations in the quartz substrate. Surface polarization caused by the vibrating quartz substrate is detected by the detection antenna, and the amplitude of the received signal at the same frequency component as the driving bursts is extracted by a heterodyne technique. Frequency range of each resonance spectrum is varied during deposition referring to the halfwidth of each peak to track the resonance peaks.

The antennas and quartz substrate were placed in the RF-magnetron sputtering chamber, and film was deposited from above the substrate. The pressure before starting deposition was less than $5.0 \times 10^{-4} \mathrm{~Pa}$, and the Ar pressure during sputtering was $0.4 \mathrm{~Pa}$. The sputtering rate was deduced from the final film thickness determined by the $\mathrm{x}$-ray reflectivity measurement and the total deposition time. Rectangularparallelepiped Z-cut monocrystal quartz was used as the substrate. The substrate dimensions were $2.5,1.7$, and $0.2 \mathrm{~mm}$ in the $X, Y$, and $Z$ directions, respectively. The thin films were deposited on the $Z$ plane.

\section{RESULTS AND DISCUSSION}

Figure 2(a) shows a representative evolution of the resonance spectrum during deposition of Ag. The resonance frequency of this vibrational mode was $2.320 \mathrm{MHz}$ before deposition. The Lorentz function was fitted to the measurements near the peak, and the resonance frequency, $f$, and the internal friction, $Q^{-1}$, were determined from the center frequency and half maximum full-width, respectively. Figures 2(b) and 2(c) show evolutions of the $f$ and $Q^{-1}$ of three different resonance vibrations, respectively. The points in Fig. 2(b) indicate the times when the resonance spectra of $2.320 \mathrm{MHz}$ in Fig. 2(a) were obtained. After the pre-sputtering procedure, deposition was started at $0 \mathrm{~s}$ by opening a shutter. The shutter was closed at 3600 s. Shortly
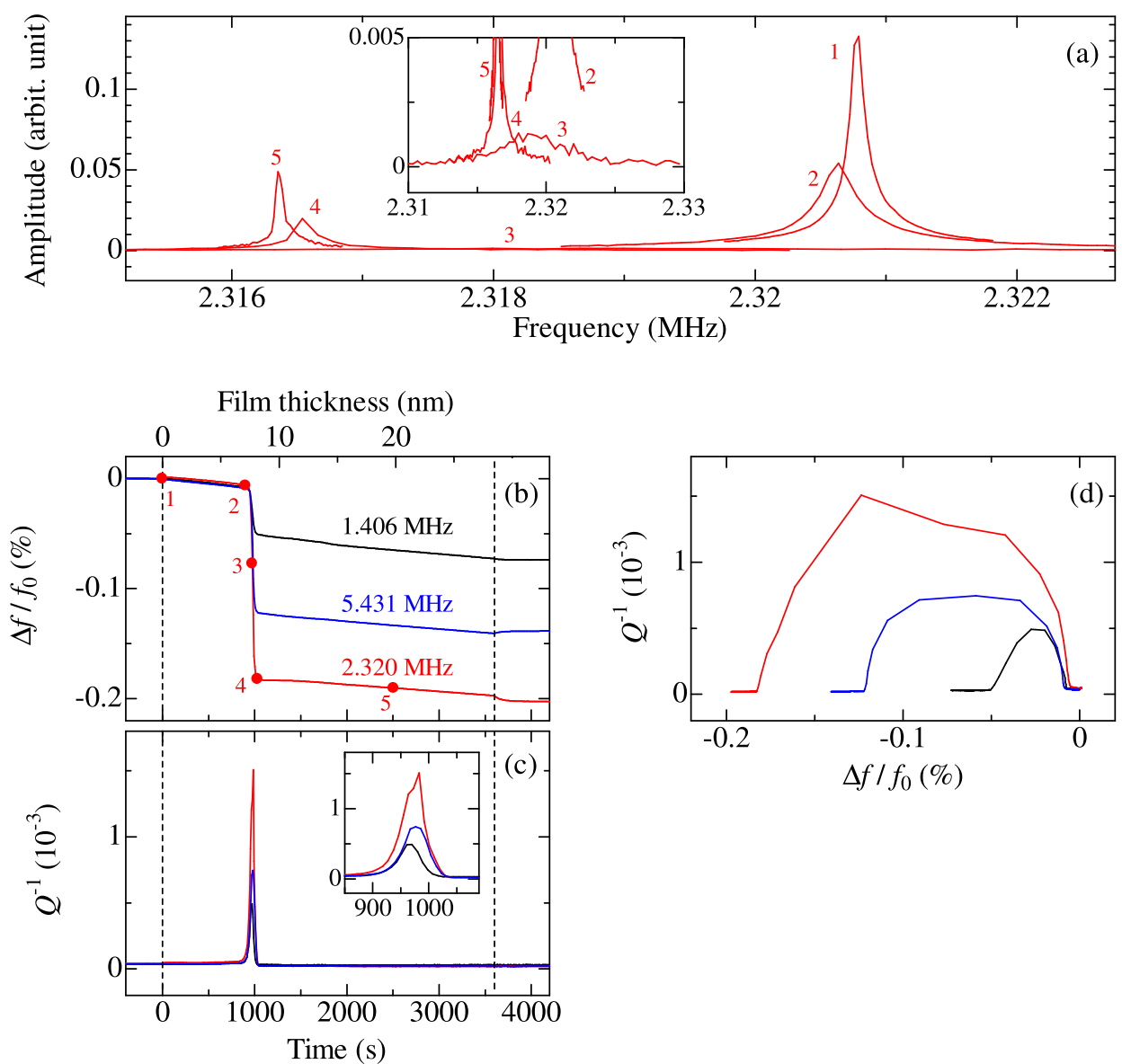

FIG. 2. Evolution of resonance properties of a quartz substrate during deposition of Ag. (a) Resonance spectra measured during deposition. Inset shows the enlarged figure. Evolutions of (b) resonance frequency and (c) internal friction. Points in (b) indicate the times when the spectra in (a) were obtained. Inset of (c) shows the enlarged figure. (d) Relationship between the resonance frequency and internal friction.

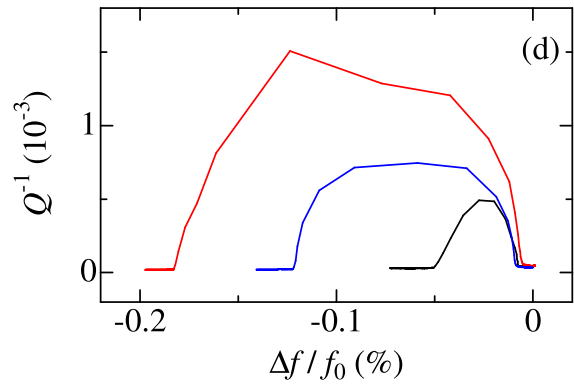


after starting the deposition, the resonance frequencies decreased almost linearly as the deposition progressed. Around $970 \mathrm{~s}$, the resonance frequencies suddenly dropped, and then continued to decrease at rates similar to those before the drop. After closing the shutter, resonance frequencies became constant.

The evolutions of the resonance frequencies are interpreted by the morphology change and accompanying resistivity change that has been observed in the previous studies, as described in the Introduction. In the present measurement, both an increase in mass and a change in conductivity should influence the resonance frequencies. Just after starting deposition, nucleation occurs and isolated islands are formed on the quartz substrate. At this stage, conductivity on the quartz surface is significantly low. Mass increment therefore is the predominant factor for frequency change; resonance frequencies decrease linearly. As the deposition progresses, the islands grow, and conductivity should increase because of the quantum-tunneling effect between islands. Further growth causes the islands to coalesce, leading to the formation of a continuous film and a drastic decrease in the resistivity. The equipotential of the film prevents the polarization at the surface of the quartz, thus weakening the apparent piezoelectricity. This weakening can explain the decrease in resonance frequency. After a continuous film has been formed, the resistance would be barely changed by deposition. Mass increment, therefore, again becomes the dominant factor for frequency change, and the resonance frequencies decrease linearly with time.

Regarding the change in internal friction, it shows a peak coincident with the drop in resonance frequency. When isolated islands are formed, hopping of carriers between them should govern the conductivity. Influence of the hopping conduction by carriers in piezoelectric semiconductors on propagating behavior of acoustic waves was investigated in the previous study ${ }^{24}$ and in SAW measurements, increase in attenuation is understood as a relaxation phenomenon with increasing conductivity; ${ }^{16}$ acoustic energy is absorbed by the hopping of carriers. We consider that this interpretation is applicable to the increase in the internal friction observed in the present study. Once a continuous structure is formed, the hopping conduction ceases to occur and internal friction decreases. In this way, $Q^{-1}$ peak appears at the transition between an island structure and a continuous film structure.

To confirm the above interpretation, morphology evolution of Ag films was observed using atomic force microscopy (AFM). Six Ag films were prepared by stopping deposition at different times for each one. Three films were prepared by stopping the deposition before the $Q^{-1}$ peak appears, corresponding to $180 \mathrm{~s}, 600 \mathrm{~s}$, and just before the peak in $Q^{-1}$. Other films were prepared by stopping deposition after the $Q^{-1}$ peak, corresponding to just after the $Q^{-1}$ peak, $2400 \mathrm{~s}$, and $3600 \mathrm{~s}$. Figures 3(a)-3(f) show the AFM images. Surface roughness $R a$ is calculated from the images, and it is plotted in Fig. 3(g). In Fig. 3(a), there are large bright particles. Considering that they are hardly observed in other films, they might be formed after rather than during deposition, but the cause is not yet known. For this film, the

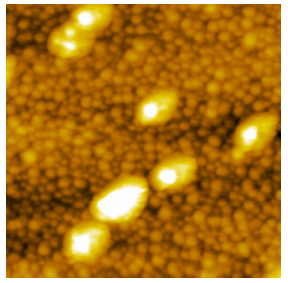

(a) $180 \mathrm{~s}$

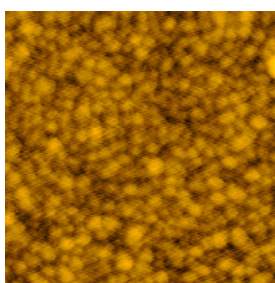

(d) After $Q^{-1}$ peak

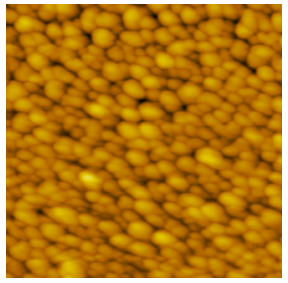

(b) $600 \mathrm{~s}$

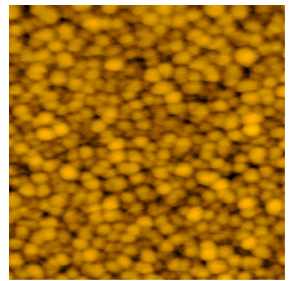

(e) $2400 \mathrm{~s}$

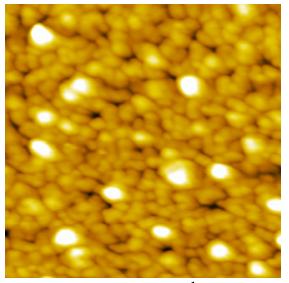

(c) Before $Q^{-1}$ peak

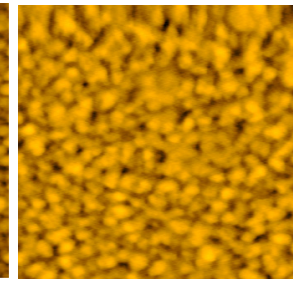

(f) $3600 \mathrm{~s}$

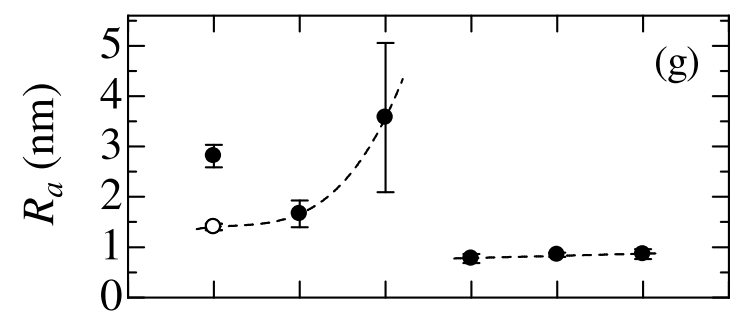

(a) (b) (c) (d) (e) (f)

FIG. 3. (a)-(f) AFM images obtained for Ag films, and (g) surface roughness. Image size is $500 \mathrm{~nm} \times 500 \mathrm{~nm}$. Surface roughness of (a) is calculated for two cases: bright large features are included (filled circle) and excluded (open circle). Dashed lines in (g) are eye guides.

surface roughness is calculated for two cases: including and excluding the large particles. The latter one is adopted in the following discussion on morphology evolution during deposition. In all images, island structure is observed. Before the $Q^{-1}$ peak, islands become larger, as the deposition progresses, increasing the surface roughness. This evolution indicates that island growth is dominant during this period. On the other hand, after the $Q^{-1}$ peak, the diameter of the islands is almost constant, and surface roughness is smaller than prior to the $Q^{-1}$ peak. These results indicate that a continuous film has been formed and islands growth in the in-plane direction is restricted. Thus, the AFM images support the interpretation that peak in $Q^{-1}$ indicates the formation of a continuous film. The transition thickness of $\mathrm{Ag}$ film, $\sim 7 \mathrm{~nm}$, is comparable with those obtained from resistivity measurements, $6-14 \mathrm{~nm},{ }^{5}$ which also supports the view that the $Q^{-1}$ peak occurs at the transition.

We here investigate the morphology change before and after the $Q^{-1}$ peak in more detail. Deposition is interrupted just before and after the $Q^{-1}$ peak, and subsequent evolutions in the resonance frequency and $Q^{-1}$ are monitored. Figure 4 shows the results. When deposition is interrupted just before the $Q^{-1}$ peak, $Q^{-1}$ decreases and resonance frequency increases. On the other hand, when deposition is stopped just after the $Q^{-1}$ peak, both $Q^{-1}$ and resonance frequency decrease. Relationships between the resonance frequency and $Q^{-1}$ are plotted in Figs. 4(c) and 4(f). When Ag is deposited continuously, the relationship shows an arch shape as 

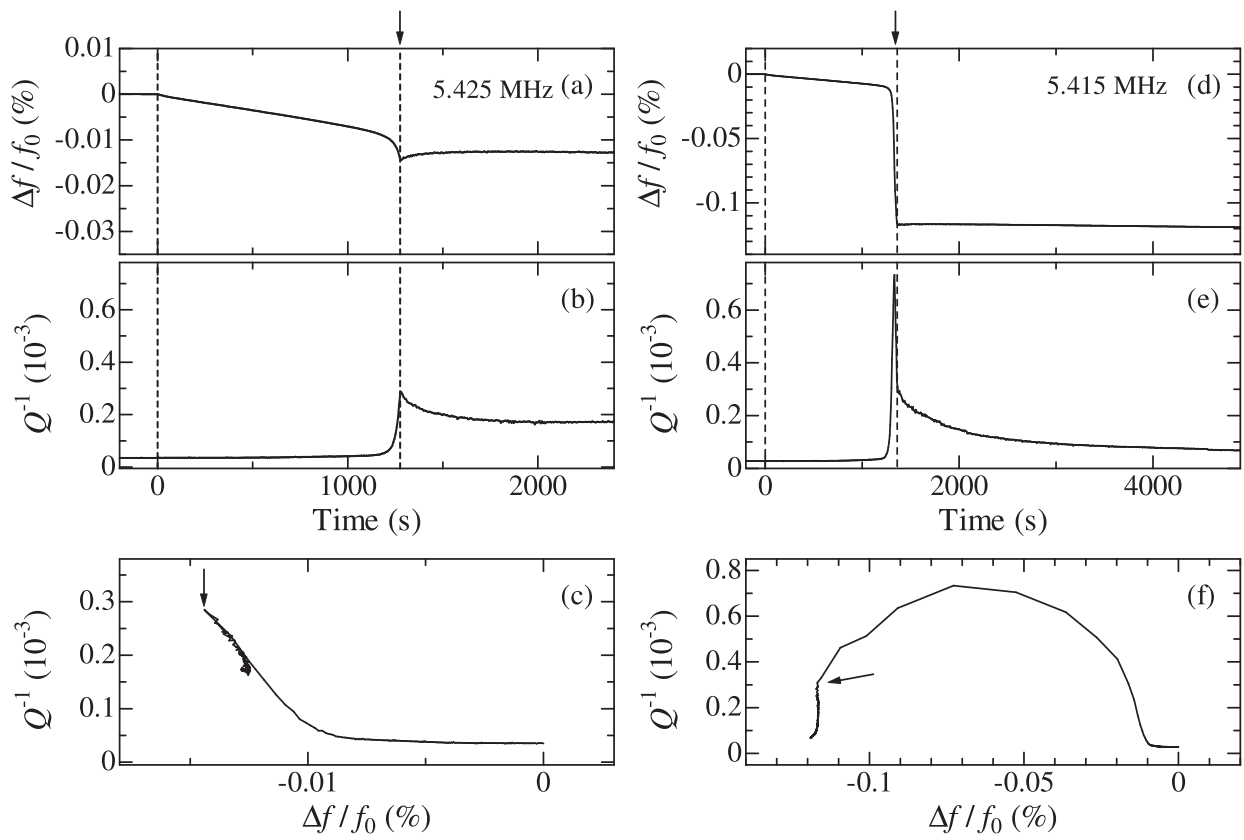

FIG. 4. Evolutions of resonance frequency and internal friction for $\mathrm{Ag}$ films obtained by interrupting the deposition (a)-(c) before and (d)-(f) after the $Q^{-1}$ peak. Deposition is interrupted by closing the shutter at which the arrow indicates. shown in Fig. 2(d); the drop in resonance frequency and the increment of $Q^{-1}$ occur simultaneously, and the transformation from discontinuous to continuous structure progresses on the arch. When the deposition interruption is taken before the $Q^{-1}$ peak, the plot goes back almost following the same path as that of the past deposition (Fig. 4(c)). By contrast, when the deposition interruption is taken after the $Q^{-1}$ peak, the plot moves forward by following the trajectory observed in the continuous deposition (Fig. 4(f)). These results indicate that films are unstable just after interrupting deposition. The former result indicates that the sputtered metal tends to form an isolated island (discontinuous) structure before the $Q^{-1}$ peak. The latter indicates that the sputtered metal tends to form a continuous film after the $Q^{-1}$ peak. Similar behavior was observed in the electric resistance measurement; ${ }^{5,6}$ resistance change during interruption turns from increment to decrement as film thickness at which the deposition is interrupted increases. The increase in resistivity during the interruption is explained by the shape change of the isolated islands from ellipsoidal to spherical to reduce their surface energy; ${ }^{8}$ the shape change makes the inter-island distance larger, and conductivity by the quantum tunneling is reduced. On the other hand, after the $Q^{-1}$ peak, a continuous film has been formed, and decrease in surface roughness can decrease the surface energy. Therefore, the surface roughness tends to be smaller. These interruption measurements, thus, confirm that the $Q^{-1}$ peak is the threshold between discontinuous and continuous structure.

The film growth monitoring was performed also for $\mathrm{Cu}$ and Pt. Results of continuous depositions and interruption measurements are shown in Figs. 5 and 6. In the continuous deposition, both $\mathrm{Cu}$ and $\mathrm{Pt}$ showed the drop of resonance frequency and peak of internal friction. These results are explained by the island growth followed by formation of a continuous film, as in the Ag film. On the other hand, somewhat different behaviors were observed in the interruption measurements. In the $\mathrm{Cu}$ film, relationship between $f$ and $Q^{-1}$ goes back by following the same path as that of the past deposition after the interruption before the $Q^{-1}$ peak. This is similar to that of Ag film. However, in the Pt film, when the deposition is interrupted just before the $Q^{-1}$ peak at (i) in Fig. 6, the resonance frequency continues to decrease, and the $Q^{-1}$ increases with time. The relationship between the frequency and $Q^{-1}$ goes forward by following the relationship observed in the continuous deposition. This result indicates that isolated $\mathrm{Pt}$ islands tend to form a continuous film when deposition is interrupted prior to the formation of a continuous film. This is an opposite trend to that observed in $\mathrm{Ag}$ and $\mathrm{Cu}$ films. After deposition is restarted by opening the shutter at (ii), the relationship goes following the arch. The shutter is closed again at (iii) after the $Q^{-1}$ peak. Then, progress of the relationship as observed in $\mathrm{Ag}$ and $\mathrm{Cu}$ is observed; the relationship continues to go following the arch.
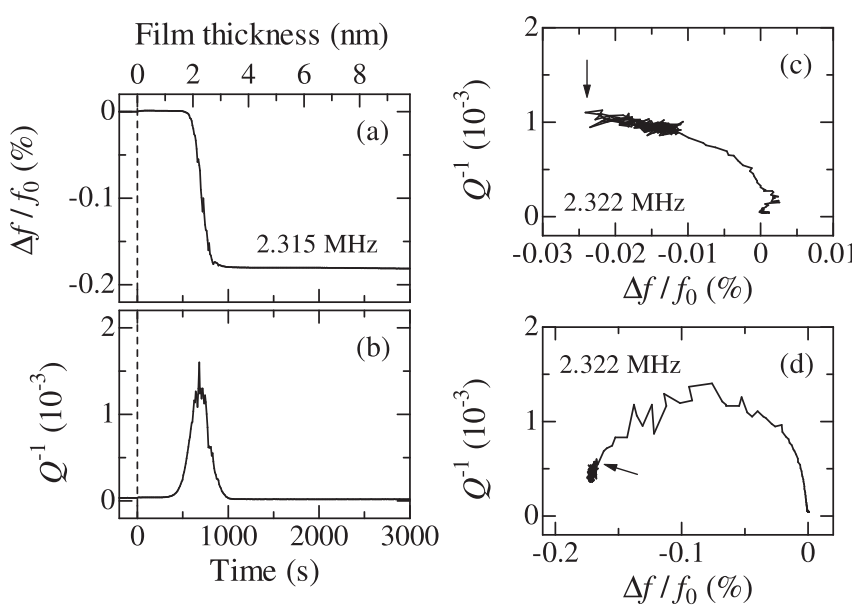

FIG. 5. Evolutions of resonance frequency and internal friction for $\mathrm{Cu}$ films obtained by (a), (b) continuous deposition, (c) interruption before the $Q^{-1}$ peak, and (d) interruption after the $Q^{-1}$ peak. The arrows indicate the time when the deposition is interrupted. 

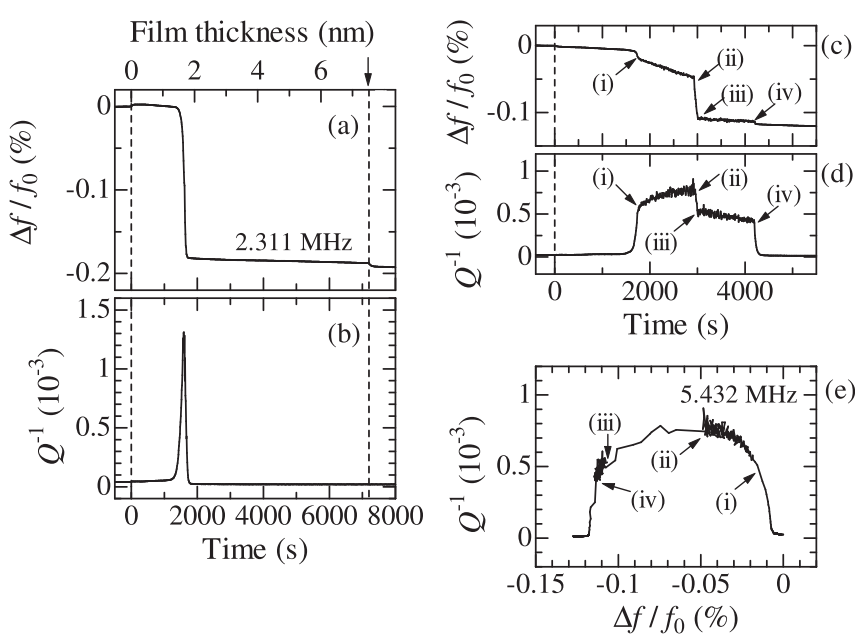

FIG. 6. Evolutions of resonance frequency and internal friction for Pt films obtained by (a), (b) continuous, and (c)-(e) interruption deposition. In (c)-(e), the deposition was interrupted at (i) and (iii) and was restarted at (ii) and (iv).

Among $\mathrm{Ag}, \mathrm{Cu}$, and $\mathrm{Pt}, \mathrm{Pt}$ has the largest surface energy: the surface energies of the (111) face of $\mathrm{Ag}, \mathrm{Cu}$, and Pt are 620,1170 , and $1440 \mathrm{ergs} / \mathrm{cm}^{2}$, respectively. ${ }^{25}$ If the morphology changes were driven by a consequent decrease in the surface energy, we would have expected Pt to show the behavior as $\mathrm{Ag}$ and $\mathrm{Cu}$. However, $\mathrm{Pt}$ showed different behaviors. Considering that frequency drop and $Q^{-1}$ peak are observed in continuous deposition of $\mathrm{Pt}$, continuous film is formed after islands growth and their percolation. Therefore, evolutions of resonance frequency and $Q^{-1}$ during interrupting the deposition before the $Q^{-1}$ peak are expected to be caused by shape change of isolated islands; spreading of $\mathrm{Pt}$ islands on the quartz substrate decreases the inter-island distance, thus increasing the conductivity. A similar shape change caused by low interfacial energy was observed in $\mathrm{Pt}$ islands on a silica substrate. ${ }^{26}$ We consider that the lower interfacial energy between $\mathrm{Pt}$ and quartz is a possible cause of the unusual behaviors we observed so far.

Finally, sensitivity of mechanical free vibration of a piezoelectric material to deposition of metallic film is described. In Fig. 2, a continuous Ag film is formed with a thickness around $7 \mathrm{~nm}$, and a significant increase of $Q^{-1}$ occurs at the same time. At that time, the ratio of film thickness to substrate thickness is about 0.000035 . It is notable that such a thin metallic film can significantly change the $Q^{-1}$ of a quartz substrate. When $10 \mu$ m-thick quartz substrate was used for the experiment, the resonance peaks disappeared during deposition, and subsequent monitoring of the resonance vibrations was not possible even after a continuous film is formed. A continuous metallic film must have prevented polarization of the small quartz substrate. Figure 7 shows resonance spectra of quartz substrates of 10 and $200 \mu \mathrm{m}$ thick before and after deposition of Ag films of about $40 \mathrm{~nm}$ thick, thick enough for the Ag films to have continuous structure. It confirms that resonance peaks of $10 \mu \mathrm{m}$-thick quartz substrate disappear after the transition from discontinuous to continuous structure. These results demonstrate that mechanical free vibration of a piezoelectric material excited by the antennas is

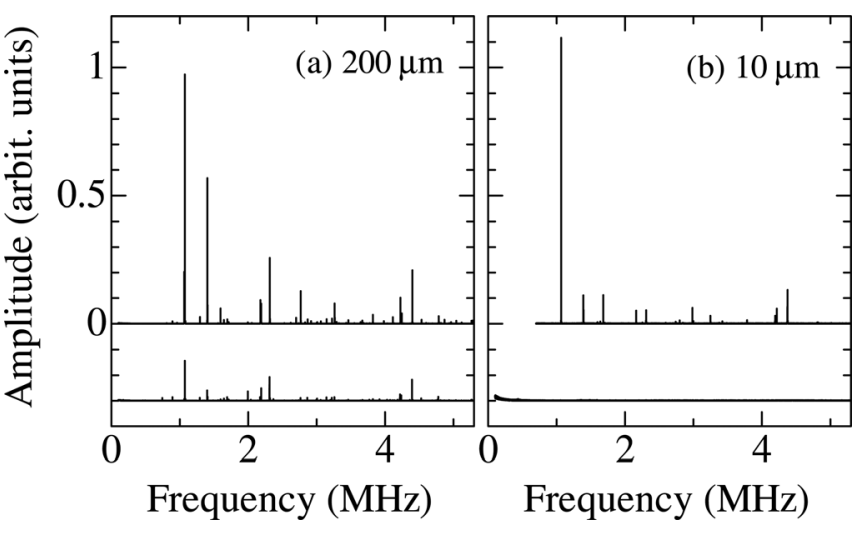

FIG. 7. Resonance spectra of quartz substrates of (a) 200 and (b) $10 \mu \mathrm{m}$ thick. Top and bottom spectra were measured before and after deposition of $\mathrm{Ag}$, respectively. The bottom spectra are shifted in the vertical axis by -0.3 .

sensitive to the deposition of a metallic film and can be a powerful method for evaluating the structural evolution of metallic films during deposition.

\section{CONCLUSIONS}

We developed the electrodeless resonance method for monitoring the growth of metallic films on quartz substrates. From the relationship between resonance frequency and internal friction, morphology change from discontinuous to continuous structure was detected during deposition. Before internal friction peak appears, metallic films tend to be composed of isolated islands, and after internal friction peak appears, they tend to be a continuous film to reduce the surface energy. In Pt films, isolated islands tend to spread on quartz, and unique behavior was observed. A possible cause of this is the low interfacial energy of Pt on quartz.

In this paper, the developed method was applied to the film-growth monitoring on piezoelectric substrates. However, by depositing buffer materials on the quartz substrates before depositing the metallic films, it will be possible to monitor film growth on materials that are not themselves piezoelectric.

\section{ACKNOWLEDGMENTS}

The authors thank Katharine E. Jensen for valuable comments on this paper.

${ }^{1}$ A. I. Maaroof and B. L. Evans, J. Appl. Phys. 76, 1047 (1994).

${ }^{2}$ T. Shima, K. Takanashi, Y. K. Takahashi, and K. Hono, Appl. Phys. Lett. 81, 1050 (2002).

${ }^{3}$ N. Nakamura, Y. Kake, H. Ogi, and M. Hirao, J. Appl. Phys. 108, 043525 (2010).

${ }^{4}$ N. Nakamura, A. Uranishi, M. Wakita, H. Ogi, M. Hirao, and M. Nishiyama, Appl. Phys. Lett. 98, 101911 (2011).

${ }^{5}$ E. Byon, T. W. H. Oates, and A. Anders, Appl. Phys. Lett. 82, 1634 (2003).

${ }^{6}$ T. W. H. Oates, L. Ryves, and M. M. M. Bilek, Opt. Express 15, 15987 (2007).

${ }^{7}$ C. A. Neugebauer and M. B. Webb, J. Appl. Phys. 33, 74 (1962).

${ }^{8}$ M. Nishiura and A. Kinbara, Thin Solid Films 24, 79 (1974).

${ }^{9}$ I. M. Rycroft and B. L. Evans, Thin Solid Films 290-291, 283 (1996).

${ }^{10}$ E. V. Barnat, D. Nagakura, P.-I. Wang, and T.-M. Lu, J. Appl. Phys. 91, 1667 (2002).

${ }^{11}$ R. Abermann, R. Kramer, and J. Mäser, Thin Solid Films 52, 215 (1978). 
${ }^{12}$ D. Winau, R. Koch, A. Führmann, and K. H. Rieder, J. Appl. Phys. 70, 3081 (1991).

${ }^{13}$ A. L. Shull and F. Spaepen, J. Appl. Phys. 80, 6243 (1996).

${ }^{14}$ J. A. Floro, S. J. Hearne, J. A. Hunter, P. Kotula, E. Chason, S. C. Seel, and C. V. Thompson, J. Appl. Phys. 89, 4886 (2001).

${ }^{15}$ P. Bierbaum, Appl. Phys. Lett. 21, 595 (1972).

${ }^{16} \mathrm{P}$. Bierbaum, J. Acoust. Soc. Am. 55, 766 (1974).

${ }^{17}$ R. Wiegert, H. Yoshida, K. J. Sun, M. Levy, H. Salvo, Jr., and B. R. Mcavoy, J. Phys. Colloq. 46, C10-737 (1985).

${ }^{18}$ M. Takahashi, H. Shoji, and M. Tsunoda, J. Magn. Magn. Mater. 134, 403 (1994).
${ }^{19}$ G. Sauerbrey, Z. Phys. 155, 206 (1959).

${ }^{20}$ K. A. Marx, Biomacromolecules 4, 1099 (2003).

${ }^{21}$ H. Ogi, Proc. Jap. Acad., Ser. B 89, 401 (2013).

${ }^{22}$ H. Ogi, K. Motohisa, T. Matsumoto, K. Hatanaka, and M. Hirao, Anal. Chem. 78, 6903 (2006).

${ }^{23}$ N. Nakamura, M. Sakamoto, H. Ogi, and M. Hirao, Rev. Sci. Instrum. 83, 073901 (2012).

${ }^{24}$ A. R. Hutson and D. L. White, J. Appl. Phys. 33, 40 (1962).

${ }^{25}$ S. M. Foiles, M. I. Baskes, and M. S. Daw, Phys. Rev. B 33, 7983 (1986).

${ }^{26}$ R. Yu, H. Song, X.-F. Zhang, and P. Yang, J. Phys. Chem. B 109, 6940 (2005). 